“ (C) 2017 IEEE. Personal use of this material is permitted. Permission from IEEE must be obtained for all other uses, in any current or future media, including

reprinting/republishing this material for advertising or promotional purposes, creating new collective works, for resale or redistribution to servers or lists, or reuse of any copyrighted component of this work in other works." 


\title{
Efficient Spatio-temporal Sensor Deployments: A Smart Building Application*
}

\author{
Linh Nguyen ${ }^{1}$, Guoqiang $\mathrm{Hu}^{1}$ and Costas J. Spanos ${ }^{2}$
}

\begin{abstract}
The paper addresses the problem of efficiently deploying sensors in spatial environments, e.g. smart buildings, for the purpose of monitoring environmental phenomena. By modelling the environmental fields using spatio-temporal Gaussian processes, a new and efficient optimality criterion of minimizing prediction uncertainties is proposed to find the best sensor locations. Though the environmental processes spatially and temporally vary, the proposed approach of choosing sensor positions is not affected by time variations, which significantly reduces computational complexity of the optimization problem. The sensor deployment problem is then solved by a practically and feasibly polynomial algorithm, where its solutions are guaranteed. The proposed approaches were implemented in a real tested space in a university building, where the obtained results are highly promising.
\end{abstract}

\section{INTRODUCTION}

In buildings, there are the facts that at least $30 \%$ of energy consumption of heating, ventilating, and air conditioning (HVAC) systems, which account for up to $47 \%$ of the building energy, are wasted [1], and that there are more and more demands on standards for indoor environmental qualities in building spaces [2]. Therefore, reducing the energy consumption and increasing the human comfort are paramount to economically and socially justify smart buildings. While the human comfort indexes are based on the indoor spatial fields such as air temperature, relative humidity, and carbon dioxide, it is technically required to optimally control the indoor environments. To this end, deeply understanding of all environmental parameters is essential for efficient energy managements of the buildings. With developments in technologies of micro-electro-mechanical systems and wireless communications, which contain the substantial evolution in reducing sizes and costs of components, a wireless sensor network (WSN) [3] empowers itself to be increasingly useful in crucial applications, especially in observing indoor environmental parameters in secondary schools' classrooms [4] and in university buildings [2], monitoring room temperature in offices [5], and detecting fire in buildings [6].

Nonetheless, in the context of deploying sensors, multiple wireless sensors can be co-located within the vicinity of a phenomenon and generate similar data samples, which apparently produces sizable redundancy in the measured

*This work was supported by the Building and Construction Authority of Singapore under EIRP-BEE Grant NRF2013EWT-EIRP004-051.

${ }^{1}$ School of Electrical and Electronic Engineering, Nanyang Technological University, Singapore linh.v.nguyendieee.org, gqhulatu.edu.sg

${ }^{2}$ Department of Electrical Engineering and Computer Science, University of California, Berkeley, CA 94720, United States spanos@berkeley .edu data. The redundant measurements have an adverse influence on effectively using the WSN since they do not provide any additional information about the observed spatial field. Moreover, the expendable samples result in many issues for a resource-constrained network of wireless sensors in terms of collecting and analyzing data, particularly in long-term monitoring.

In the literature, the work [7] developed deployment criterion that relies on observability and controllability Gramians, where objective of finding optimal sensor locations is to improve performances of the HVAC systems. In another work [8] Brunelli et al. intentionally positioned sensors at working areas in a university department to observe indoor environments. However, the sensor locations are not optimal and the obtained results do not present spatial distributions of the indoor environmental parameters in the whole space. The authors in [9] proposed to separate sensor nodes into clusters where sensors have the same output signals, and one sensor in each cluster is then representative of the whole cluster.

Though there are some approaches proposed for the sensor deployments in the buildings, their metrics of finding sensor locations do not address the quality of sensing as well as the prediction accuracy. In fact, questions of 1) how many sensors is to be needed to observe an environmental field in a specific building space and 2) where to effectively locate them are really problematic. Particularly, the challenge is to minimize the number of wireless sensors to be utilized but maximize the information collected so that prediction accuracy of a environment map is guaranteed by a desired threshold. In equivalent words, given the number of sensors, it is required to find sensor locations in the building space in order to minimize prediction uncertainties. All these issues are fully addressed in this work.

The rest of the paper is arranged as follows. Sensor deployment problem for environmental monitoring is stated in Section II. Section III presents a separable approach before it is evaluated in a real sensor deployment scenario in Section IV. Conclusions of the work are delineated in Section V. For reasons of space, proofs are omitted.

\section{Sensor Deployment Problem}

As introduced, monitoring indoor environments is a paramount task that is required to be completed before any control strategies can be carried out. What people are usually concerned about in environmental monitoring is sensing quality. In other words, given a number of observations, a map of a physical parameter in whole space can be created by predicting the field at unmeasured points, and 
then uncertainties at all predicted locations are desired to be minimal.

We consider a network of $n$ wireless sensors whose locations are denoted as $\mathbf{s}=\left(\mathbf{s}_{1}^{T}, \mathbf{s}_{2}^{T}, \cdots, \mathbf{s}_{n}^{T}\right)^{T} \in \mathbb{R}^{d \times n}$. Then collective measurements gathered by the network during the time $\mathbf{t}=\left(t_{1}, t_{2}, \cdots, t_{m}\right)^{T} \in \mathbb{R}^{m}$ can be described by the Gaussian process (GP) [10] as follows.

$$
Y(\mathbf{s}, \mathbf{t}) \sim \mathcal{N}(\mu, \Sigma),
$$

where $\mu$ is a scalar value that is a mean of all available observations. $\Sigma$ is the $n m \times n m$ covariance matrix of $Y(\mathbf{s}, \mathbf{t})$. Now, let $N$ and $M$ denote a number of unobserved locations of interest in the space and a number of points in the time to be predicted, respectively. Here $N \gg n$. According to the marginalization property of the GP [11], the posterior covariance matrix of random variables $Z\left(\mathbf{s}_{N}, \mathbf{t}_{M}\right)$ at predicted spatio-temporal locations $\left(\mathbf{s}_{N}, \mathbf{t}_{M}\right)$ given the observations $Y(\mathbf{s}, \mathbf{t})$ is computed by

$$
\Sigma_{Z\left(\mathbf{s}_{N}, \mathbf{t}_{M}\right) \mid Y(\mathbf{s}, \mathbf{t})}=\Sigma_{Z\left(\mathbf{s}_{N}, \mathbf{t}_{M}\right)}-\Sigma_{Y Z}^{T} \Sigma^{-1} \Sigma_{Y Z},
$$

where $\Sigma_{Z\left(\mathbf{s}_{N}, \mathbf{t}_{M}\right)}$ is the covariance matrix of $Z\left(\mathbf{s}_{N}, \mathbf{t}_{M}\right)$, and $\Sigma_{Y Z}$ is the cross-covariance matrix of $Y(\mathbf{s}, \mathbf{t})$ and $Z\left(\mathbf{s}_{N}, \mathbf{t}_{M}\right)$. It can be seen that the uncertainties at predicted spatio-temporal points $\left(\mathbf{s}_{N}, \mathbf{t}_{M}\right)$ are on the diagonal line of the posterior covariance matrix $\Sigma_{Z\left(\mathbf{s}_{N}, \mathbf{t}_{M}\right) \mid Y(\mathbf{s}, \mathbf{t})}$. Therefore, the problem of sensor deployments becomes finding $n$ locations in a room for deploying $n$ wireless sensors so that the uncertainties at $\left(\mathbf{s}_{N}, \mathbf{t}_{M}\right)$ are minimized.

In this paper, it is proposed to compute a total of the posterior variances at all predicted spatio-temporal locations $\left(\mathbf{s}_{N}, \mathbf{t}_{M}\right)$. That is, the formal formula of the proposed criterion is to calculate the trace of $\Sigma_{Z\left(\mathbf{s}_{N}, \mathbf{t}_{M}\right) \mid Y(\mathbf{s}, \mathbf{t}) \text {. Let }}$ us define $\mathcal{P}$ a set of all possible locations where wireless sensors can be deployed to observe the physical fields in a room, where $\operatorname{card}(\mathcal{P})=p$ is cardinality of $\mathcal{P}$. The sensor deployment is to address the problem of choosing a subset $\mathcal{C} \subseteq \mathcal{P}$, where $\operatorname{card}(\mathcal{C})=n$, so that if $n$ wireless sensors are positioned at $n$ locations in $\mathcal{C}$ then the corresponding measurements $Y(\mathbf{s}, \mathbf{t})$ allow the total of the posterior variances at $\left(\mathbf{s}_{N}, \mathbf{t}_{M}\right)$ to be minimized. Mathematically, the sensor deployment problem is initially formulated as follows.

$$
\begin{gathered}
\mathcal{C}^{o p t}=\underset{\mathcal{C} \subseteq \mathcal{P}}{\operatorname{argmin}} \operatorname{tr}\left(\Sigma_{Z\left(\mathbf{s}_{N}, \mathbf{t}_{M}\right) \mid Y(\mathbf{s}, \mathbf{t})}\right), \\
\operatorname{card}(\mathcal{C})=n
\end{gathered}
$$

where $\mathcal{C}^{o p t}$ is the optimal set of sensor locations. It can be clearly seen that $\Sigma_{Z\left(\mathbf{s}_{N}, \mathbf{t}_{M}\right)}$ in (2) is not dependent on $\mathcal{C}$. Consequently, the problem (3) can be simplified by

$$
\begin{aligned}
& \mathcal{C}^{o p t}=\operatorname{argmax} \operatorname{tr}\left(\Sigma_{Y Z}^{T} \Sigma^{-1} \Sigma_{Y Z}\right) . \\
& \mathcal{C} \subseteq \mathcal{P} \\
& \operatorname{card}(\mathcal{C})=n
\end{aligned}
$$

It is to be noted that (4) is a combinatorial optimization problem. Choosing a subset $\mathcal{C}$ out of a possible set $\mathcal{P}$ in the combinatorial optimization problem is always NP-hard [12]. Nevertheless, up to now, the NP-hard problem can be nearoptimally solved by an approximate polynomial algorithm called the greedy algorithm. Let us consider how the greedy method can deal with the NP-hard problem in (4). Obviously, cost of computing $\Sigma_{Y Z}^{T} \Sigma^{-1} \Sigma_{Y Z}$ requires $\mathcal{O}\left(N^{2} M^{2} n m\right)$ operations. Then the greedy algorithm can approximately address the problem in (4) with $\mathcal{O}\left(N^{2} M^{2} n^{2} m p\right)$ complex requirement [11]. Moreover $N \gg n$, which leads to the fact that the criterion in (4) is computationally costly. Thus, further simplification is needed. By the use of the properties of trace of a matrix, where $\operatorname{tr}(A B)=\operatorname{tr}(B A)$, the problem of sensor deployments is eventually defined as follows.

$$
\begin{gathered}
\mathcal{C}^{o p t}=\underset{\mathcal{C} \subseteq \mathcal{P}}{\operatorname{argmax}} \operatorname{tr}\left(\Sigma_{Y Z} \Sigma_{Y Z}^{T} \Sigma^{-1}\right), \\
\operatorname{card}(\mathcal{C})=n
\end{gathered}
$$

where complexity of computing $\Sigma_{Y Z} \Sigma_{Y Z}^{T} \Sigma^{-1}$ is $\mathcal{O}\left(N M n^{2} m^{2}\right)$.

It is apparent that if the issue in (5) is comprehensively solved, the question 2) in Section of Introduction is answered. Furthermore, the solutions of the problem in (5) imply that by varying the number of sensors until the total of the posterior variances at all predicted spatio-temporal locations $\left(\mathbf{s}_{N}, \mathbf{t}_{M}\right)$ satisfies a predefined requirement, the number of sensors in that case is an answer to the question 1 ) in the same paragraph.

\section{A SEPARABLE APPROACH}

It can be noticed that the problem stated in (5) can be practically resolved in a small-scale sensor network with a small data set. The problem definitely becomes intractable as it is applied to a large-scale network ( $n$ is large), where measurements are collected within a long period of time $(\mathrm{m}$ is large). In this section, we present a separable method to reduce the complexity of the problem of sensor deployments.

In this approach, a spatio-temporal separable correlation function [10] is proposed to be employed in the space-time field model. If dependence between the data is separable in terms of space and time, the covariance matrix $\Sigma$ of the collective measurements $Y(\mathbf{s}, \mathbf{t})$ can be represented by block structures. In other words, $\Sigma$ is delineated by a Kronecker product as follows.

$$
\Sigma=\Sigma^{(s)} \otimes \Sigma^{(t)}
$$

where $\Sigma^{(s)}$ is a $n \times n$ covariance matrix of purely spatial covariance values, and $\Sigma^{(t)}$ is a $m \times m$ covariance matrix of purely temporal covariance values.

Due to restructuring the covariance matrix of all available observations, the criterion for the problem of sensor deployments in buildings (5) can be simplified as the following.

Theorem 1: If a correlation function of a spatio-temporal environmental field model is separable, the problem of deploying sensors in buildings to observe indoor spatiotemporal fields is only dependent on space variations, not time variations. 
The optimality criterion of the sensor deployment problem can be now spatially stated as follows.

$$
\begin{gathered}
\mathcal{C}^{o p t}=\underset{\substack{\mathcal{C} \subseteq \mathcal{P}\\
}}{\operatorname{card}(\mathcal{C})=n} \operatorname{tr}\left(\Sigma_{Y Z}^{(s)}\left(\Sigma_{Y Z}^{(s)}\right)^{T}\left(\Sigma^{(s)}\right)^{-1}\right) .
\end{gathered}
$$

Complexity of computing $\Sigma_{Y Z}^{(s)}\left(\Sigma_{Y Z}^{(s)}\right)^{T}\left(\Sigma^{(s)}\right)^{-1}$ in (7) is $\mathcal{O}\left(N n^{2}\right)$, which is significantly lower than that in (5). In the following, we present how the greedy algorithm nearoptimally addresses the issue in (7). Let us define a nearoptimal subset corresponding to $\mathcal{C}^{o p t}$ as $\mathcal{C}^{n-o p t}$.

It is assumed that at the beginning, the near-optimal subset is empty, $\mathcal{C}^{n-o p t}=\oslash$. The algorithm randomly chooses a point $\mathbf{s}_{i}, i=1, \cdots, p$, from the possible set $\mathcal{P}, \mathbf{s}_{i} \in \mathcal{P}$. It is also supposed that the corresponding measurement at $\mathbf{s}_{i}$ is $y\left(\mathbf{s}_{i}\right) . Y(\mathbf{s}, \mathbf{t})=Y(\mathbf{s})=\left\{y\left(\mathbf{s}_{i}\right)\right\}$ and $Z\left(\mathbf{s}_{N}, \mathbf{t}_{M}\right)=Z\left(\mathbf{s}_{N}\right)$ as time is no longer involved in the calculation. It computes $\Sigma_{Y Z}^{(s)}\left(\Sigma_{Y Z}^{(s)}\right)^{T}\left(\Sigma^{(s)}\right)^{-1}$, then it iterates the computations for each other location $\mathbf{s}_{i} \in \mathcal{P}$. Each calculation returns one real value. A sequence of obtained values consequently corresponds to the possible set $\mathcal{P}$. Choosing the maximum value from this sequence, it can find the corresponding location from $\mathcal{P}$. This chosen point is the first near-optimal sensor location, denoted as $\mathbf{s}_{1}^{n-o p t}$, and $\mathcal{C}^{n-o p t}=\left\{\mathbf{s}_{1}^{n-o p t}\right\}$. Correspondingly, $Y(\mathbf{s})$ now firmly has $y\left(\mathbf{s}_{1}^{n-o p t}\right), Y(\mathbf{s})=\left\{y\left(\mathbf{s}_{1}^{n-o p t}\right)\right\}$. The chosen location $\mathbf{s}_{1}^{n-o p t}$ is now removed from $\mathcal{P}$. In the next step, the algorithm again chooses a location $\mathbf{s}_{i}, i=1, \cdots, p-1$, from the remaining $\mathcal{P}$ and temporally adds it into $\mathcal{C}^{n-o p t}$, $\mathcal{C}^{n-o p t}=\left\{\mathcal{C}^{n-o p t}, \mathbf{s}_{i}\right\}$. Likewise, the corresponding measurement $y\left(\mathbf{s}_{i}\right)$ is also temporally added into $Y(\mathbf{s}), Y(\mathbf{s})=$ $\left\{Y(\mathbf{s}), y\left(\mathbf{s}_{i}\right)\right\}$. Every $\Sigma_{Y Z}^{(s)}\left(\Sigma_{Y Z}^{(s)}\right)^{T}\left(\Sigma^{(s)}\right)^{-1}$ in the second step, corresponding to one $\mathbf{s}_{i} \in \mathcal{P}$, is a $2 \times 2$ matrix, and their traces create another sequence. Finding the sequence's maximal value and choosing its corresponding location in $\mathcal{P}$, one has the second near-optimal sensor location, which is then firmly moved from $\mathcal{P}$ to $\mathcal{C}^{n-o p t} . Y(\mathbf{s})$ also has a second permanent element. Iteratively, the algorithm runs this iteration until cardinality of $\mathcal{C}^{n-o p t}$ reaches to $n$. Notice that in each iteration, a new near-optimal sensor location obtained is greedily added into $\mathcal{C}^{n-o p t}$. The greedy algorithm addressing the sensor deployment problem is illustratively summarized in Algorithm 1.

Theorem 2: The near-optimal solutions for the sensor deployment problem obtained by the greedy algorithm are guaranteed by a $1-\left(1-\frac{1}{n}\right)^{n}$ level of optimal performances, where $n$ is number of sensors to be deployed.

\section{REAL EXPERIMENTS IN SMART BUILDINGS}

In this section, we present the results of applying the proposed approach for deploying the wireless sensors at the best locations in the tested room of S2.1-B4-01, Nanyang Technological University campus.

\section{A. Near-optimal Sensor Locations}

As discussed by the Algorithm 1, it is firstly required to find all possible sensor locations in the experimental room. $\overline{\text { Algorithm } 1 \text { Approximation algorithm for sensor deploy- }}$ ments in smart buildings

\section{Input:}

1) Set of possible sensor locations $\mathcal{P}$

2) Number of sensors $n$

\section{Output:}

1) Near-optimal set of sensor locations $\mathcal{C}^{n-o p t}$

$$
\begin{aligned}
& \text { At start, do } \\
& \mathcal{C}^{n-o p t} \leftarrow \oslash \\
& \text { 1: for } i=1 \text { to } p \text { do } \\
& \text { 2: } \quad \mathbf{s}_{i} \in \mathcal{P} \\
& \text { 3: } \quad Y(\mathbf{s})=\left\{y\left(\mathbf{s}_{i}\right)\right\} \\
& \text { 4: } \quad \text { Compute } \Sigma_{Y Z}^{(s)}\left(\Sigma_{Y Z}^{(s)}\right)^{T}\left(\Sigma^{(s)}\right)^{-1} \\
& \text { 5: end for } \\
& \text { 6: } \mathbf{s}_{1}^{n-o p t} \leftarrow \underset{\mathbf{s}_{i} \in \mathcal{P}}{\operatorname{argmax}} \Sigma_{Y Z}^{(s)}\left(\Sigma_{Y Z}^{(s)}\right)^{T}\left(\Sigma^{(s)}\right)^{-1} \\
& \text { 7: } \mathcal{C}^{n-o p t}=\left\{\begin{array}{c}
\mathbf{s}_{i} \in \mathcal{P} \\
\mathbf{s}_{1}^{n-o p t}
\end{array}\right\} \\
& \text { 8: } Y(\mathbf{s})=\left\{y\left(\mathbf{s}_{1}^{n-o p t}\right)\right\} \\
& \text { 9: } \mathcal{P} \leftarrow \mathcal{P} \backslash \mathbf{s}_{1}^{n-o p t} \\
& \text { 10: for } k=2 \text { to } n \text { do } \\
& \text { 11: } \quad \mathcal{C}_{\text {tmp }}^{n-o p t}=\mathcal{C}^{n-o p t} \\
& \text { 12: } \quad Y_{t m p}(\mathbf{s})=Y(\mathbf{s}) \\
& \text { 13: for } i=1 \text { to cardinality of } \mathcal{P} \text { do } \\
& \text { 14: } \quad \mathbf{s}_{i} \in \mathcal{P} \\
& \text { 15: } \quad \mathcal{C}^{n-o p t}=\left\{\mathcal{C}_{\text {tmp }}^{n-o p t}, \mathbf{s}_{i}\right\} \\
& \text { 16: } \quad Y(\mathbf{s})=\left\{Y_{\text {tmp }}(\mathbf{s}), y\left(\mathbf{s}_{i}\right)\right\} \\
& \text { 17: } \quad \text { Compute } \operatorname{tr}\left(\Sigma_{Y Z}^{(s)}\left(\Sigma_{Y Z}^{(s)}\right)^{T}\left(\Sigma^{(s)}\right)^{-1}\right) \\
& \text { 18: end for } \\
& \text { 19: } \quad \mathbf{s}_{k}^{n-o p t} \leftarrow \underset{\mathbf{s}_{i} \in \mathcal{P}}{\operatorname{argmax}} \operatorname{tr}\left(\Sigma_{Y Z}^{(s)}\left(\Sigma_{Y Z}^{(s)}\right)^{T}\left(\Sigma^{(s)}\right)^{-1}\right) \\
& \text { 20: } \quad \mathcal{C}^{n-o p t}=\left\{\mathcal{C}_{t m p}^{n-o p t}, \mathbf{s}_{k}^{n-o p t}\right\} \\
& \text { 21: } \quad Y(\mathbf{s})=\left\{Y_{t m p}(\mathbf{s}), y\left(\mathbf{s}_{k}^{n-o p t}\right)\right\} \\
& \text { 22: } \quad \mathcal{P} \leftarrow \mathcal{P} \backslash \mathbf{s}_{k}^{n-o p t} \\
& \text { 23: end for }
\end{aligned}
$$

For the purpose of simplicity, we first discretized the room into a $100 \times 100$ grid. That is, each small spatial area of the grid is approximately sized $20 \mathrm{~cm} \times 15 \mathrm{~cm}$, which is empirically reasonable to locate our available Libelium and Monnit sensor nodes. According to the layout map of the room, all unavailable areas of cubicles, lab benches, personal computer tables, occupants' desk stations and experimental facilities are identified. We then removed all cells on the grid, which correspondingly overlap the unavailable areas, from the set of the possible points. The cells remaining on the grid are all the possible locations that can be utilized to deploy the wireless sensors. The possibility of sensor locations is visually illustrated by the blue circle points in Fig. 1.

Illustratively, measurements of the heat in the test room were collected by a network of temperature sensors every two hours in four weeks from 7 March to 3 April 2016. Hence, $Y(\mathbf{s}, \mathbf{t})$ consists of 6720 temperature values. We also assumed that $N=22500$ unmeasured positions on a grid of $150 \times 150$. By implementing the Algorithm 1 into the 




Fig. 1: Possible sensor locations (blue circles) in room S2.1B4-01, Nanyang Technological University

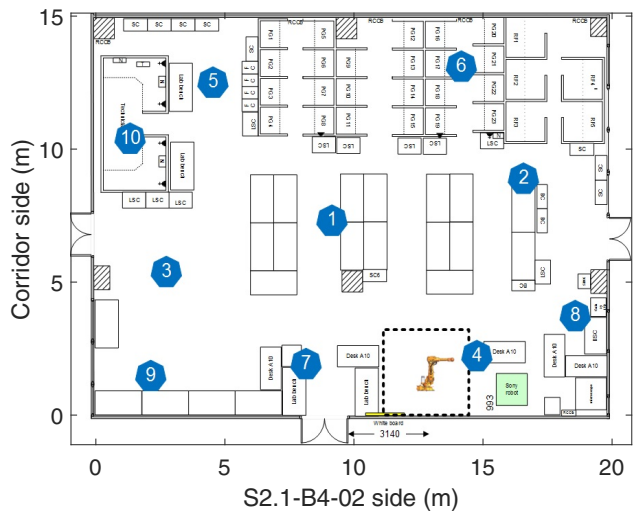

Fig. 2: 10 near-optimal sensor locations, ordered from most to least informative locations, in S2.1-B4-01 room, Nanyang Technological University

collective dataset, we near-optimally found 10 best locations, for example, for effectively deploying sensors, illustrated in Fig. 2. Note that they are numbered from most to least informative positions. In equivalent words, if we have only 5 temperature sensors, we will locate them at the positions numbered from 1 to 5 .

\section{B. Result Discussions}

After finding 10 best locations as shown in Fig. 2, since we have a limited number of sensors and we intended to employ them for the twofold purposes of training and comparing, we separated the 10 available temperature wireless sensors into two groups. More specifically, we first used 5 sensors to locate at 5 locations numbered from 1 to 5 in Fig. 2; and their measurements were employed to train a space-time model of the temperature field in the room. We also utilized 5 other sensors to position at 5 empirical locations in the room; and their observations were used to train another model for the purpose of comparisons. In fact, due to limitations of time and facilities, we could not run many random deployments in the room. Like any technicians, when they would monitor indoor spatial environments, if they do not have any methods to find the best sensor locations, they would decide where to deploy the sensors based on their experiences. Therefore, the 5 empirical locations were chosen based on the authors' point of view, which can be visually seen in Fig. 3f.

In this implementation, we deployed the wireless sensors in the tested room in two other weeks, from 25 April to 08 May 2016. By using the same settings of the wireless network configurations, each sensor also sampled the indoor temperature every two hours. Hence, each group of 5 (nearoptimal or empirical) wireless sensors collected 840 temperature values in the two studied weeks. We utilized the 420 measurements collected in the first week (25 April to 01 May 2016), corresponding to the 5 near-optimal locations, to learn a space-time temperature model that is called model $_{\text {opt }}$. We also employed the other 420 measurements of the same week, corresponding to the 5 empirical locations, to learn another spatio-temporal temperature model that is called model $_{e m p}$. Both the temperature models model $_{\text {opt }}$ and model $_{\text {emp }}$ could be then employed to predict and estimate the heat at any time and any locations in the experimented room. For instance, to validate the predictions, we utilized these two space-time models to predict the temperature fields in the room in the following week, at 11:17 on 06 May 2016. The results are demonstrated in Figures $3 \mathrm{c}$ to $3 \mathrm{f}$. More importantly, for the purpose of comparisons, a purely spatial model was also developed by the use of 10 temperature measurements gathered by the 10 available wireless sensors at the same time, $11: 17$ on 06 May 2016. This purely spatial model was then employed to predict the temperature field in the whole tested room, as illustrated in Figures $3 a$ and $3 b$.

In other words, Figures $3 \mathrm{a}, 3 \mathrm{c}$ and $3 \mathrm{e}$ are the heat maps of the experimented room at 11:17 on 06 May 2016. While Fig. 3a was created by the 10 realistic observations directly taken at the same time, the maps in Figures $3 \mathrm{c}$ and $3 \mathrm{e}$ were created by the space-time temperature models model $_{\text {opt }}$ and model $_{e m p}$ learned in one week previously, respectively. Illustratively, though there is no much difference among them, the temperature map obtained by the 5 sensors positioned at the near-optimal locations in Fig. $3 \mathrm{c}$ is more closely approximate to that obtained by all the temperature sensors in Fig. 3a. More importantly, substantial differences can be obviously seen in the prediction standard errors surfaces, as demonstrated in Figures 3b, 3d and 3f. It is apparent that the prediction error variance at every point obtained by the model model $_{\text {opt }}$ learned from the spatio-temporal measurements of the 5 near-optimal sensors in Fig. $3 \mathrm{~d}$ is quite similar to that at a corresponding point in Fig. $3 \mathrm{~b}$, which are created by the model using all the 10 sensor measurements. Nevertheless, the prediction standard errors in Fig. 3f obtained by the model model $_{e m p}$, which was trained by the observations of the temperature sensors positioned by our experience, are much higher than those in Figures $3 b$ and $3 d$, at every compared point.

Consequently, although there is a network of only 5 wireless sensors deployed at the locations found by our proposed approach to be utilized in taking the temperature measurements, the results of its spatio-temporal model in 
Mean from spacetime model learned by 10 sensors

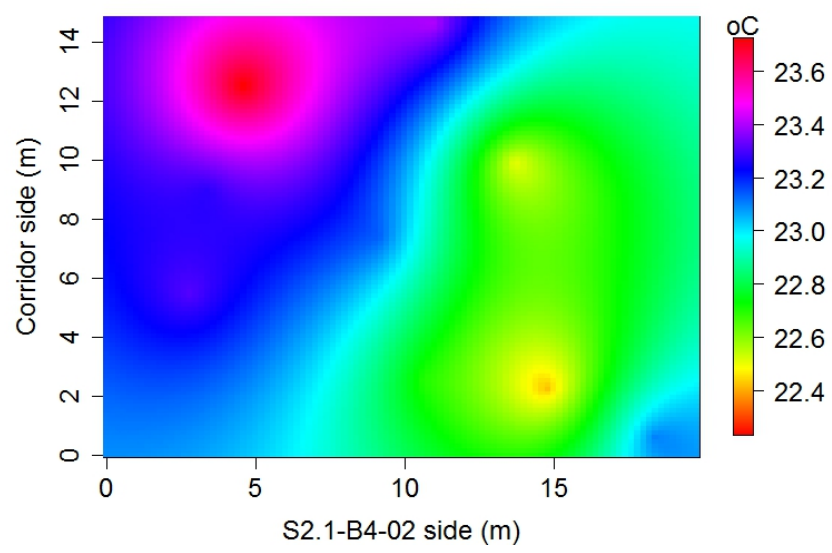

(a)

Mean from spacetime model learned by $\mathbf{5}$ selected sensors

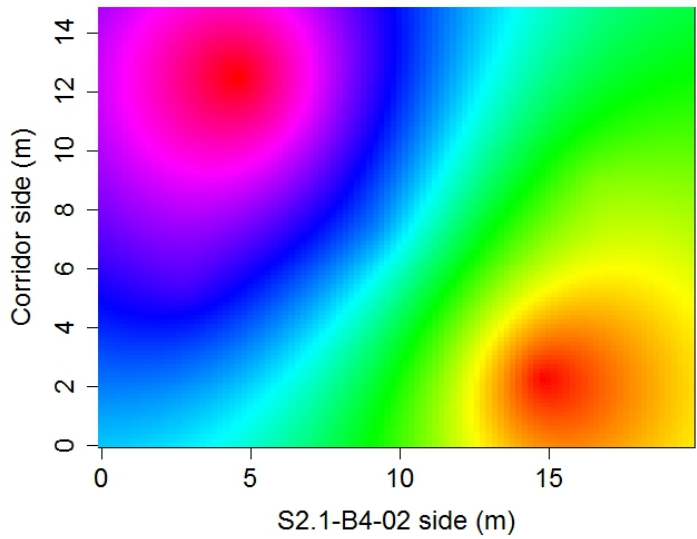

(c)

Mean from spacetime model learned by 5 empirical sensors

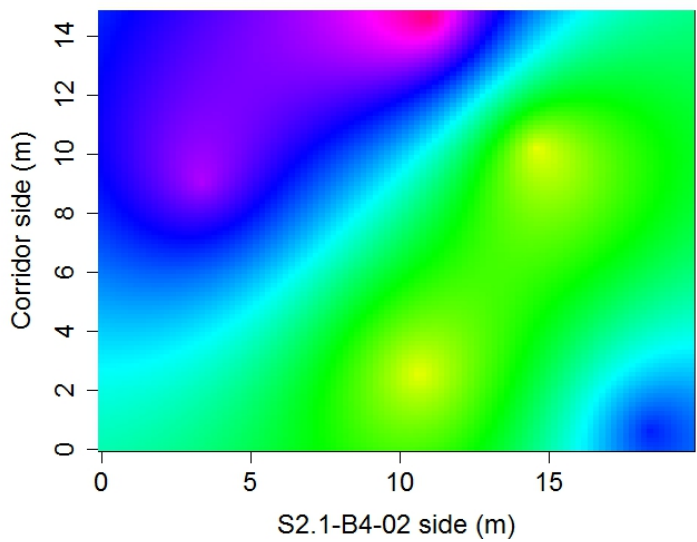

(e)

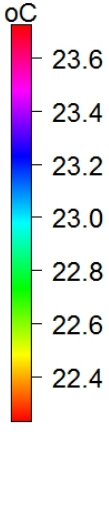

23.4

23.2

23.0

22.8

22.6

22.4

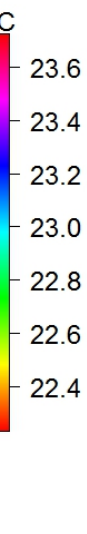

23.4

23.2

23.0

22.8

22.6

22.4

Fig. 3: Predicted temperature fields (left column) and prediction standard errors (right column) at 11:17 on 06 May 2016, obtained by a purely spatial model learned by temperature values collected by 10 sensors deployed at beginning as shown in (a) and (b), and by spatio-temporal models learned by the use of different sets of measurements gathered by: 5 sensors deployed at 5 near-optimal locations as shown in (c) and (d), and 5 sensors located empirically as shown in (e) and (f). Ranges of fields and standard errors are demonstrated in color bars. 


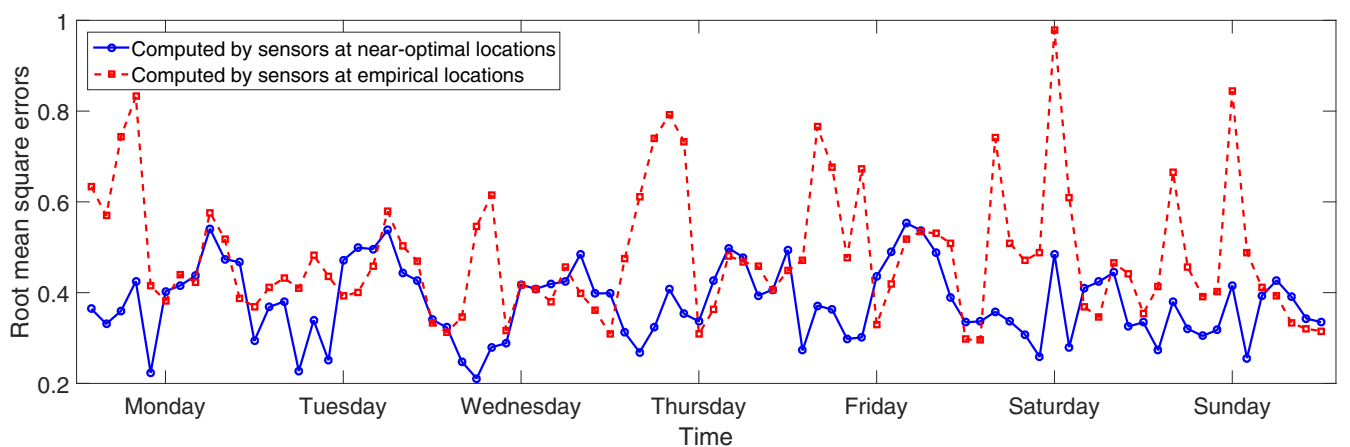

Fig. 4: Root mean square errors computed by sensors at near-optimal locations against by sensors at empirical locations over the week from 25 April to 01 May 2016.

terms of both the predicted field and the prediction standard errors are highly comparable to those obtained by a network of 10 deployed sensors. Particularly, due to the criterion of selecting the sensor locations by minimizing total variances, the proposed method results in the small errors in the predictions, which are correspondingly a half of those in the empirical approach.

Furthermore, the good quality of the predictions in our sensor deployment method was demonstrated by computing the RMSE. In the compared experiments, we employed the space-time model model ${ }_{\text {opt }}$ to predict the temperature at the empirical locations over the week from 25 April to 01 May 2016. The predicted values and the real measurements at the empirical locations were then used to calculate the RMSE, which are illustrated by the blue curve in Fig.4. On the other hand, we also utilized the spatio-temporal model model $_{e m p}$ to estimate the heat at the nea-optimal locations found by our proposed technique in the same week. The RMSE between the predictions and the realistic observations at the nearoptimal points were similarly computed and plotted by the dash red curve in Fig. 4. Generally, in Fig. 4, the blue curve is mostly lower than the dash red one. That is, the sensors deployed at the near-optimal locations have more information about all the other locations in the tested room than the sensors positioned at the empirical locations.

It can be clearly seen that the proposed approach can very efficiently address the sensor deployment problem in buildings, though its solutions are only near-optimal. However, as presented in Section III, the solutions obtained by our method are guaranteed by a bound. For instance, in this experiment, we intended to find 5 best locations for deploying the 5 temperature sensors in the S2.1-B4-01 room. By applying the proposed algorithm to solving the problem, the results are at least bounded by a level of 67.23 percents as compared with the optimal performance.

\section{Conclusions}

The paper has considered the issue of deploying sensors for monitoring indoor climate in smart buildings. Based on space-time Gaussian processes, a separable optimization approach to efficiently find the best environmental sensor locations in the building spaces was developed, which is only dependent on spatial variations, though measurements are gathered through time. The optimality problem of sensor deployments is near-optimally addressed by a greedy algorithm, yet its performances are guaranteed by a level of $1-\left(1-\frac{1}{n}\right)^{n}$ as compared with the optimum, where $n$ is the number of sensors. The effectiveness of the proposed approach was extensively evaluated in a real space in a university building.

\section{REFERENCES}

[1] Building Energy Data Book, U.S. Department of Energy, 2011.

[2] D. Brunelli, I. Minakov, R. Passerone, and M. Rossi, "Smart monitoring for sustainable and energy-efficient buildings: A case study," in Proc. IEEE Workshop on Environmental, Energy and Structural Monitoring Systems, Trento, Italy, July 2015.

[3] A. Rogers, E. David, and N. R. Jennings, "Self-organized routing for wireless microsensor networks," IEEE Transactions on Systems, Man, and Cybernetics - Part A: Systems and Humans, vol. 35(3), pp. 349359, 2005.

[4] L. D. Pereira, D. Raimondo, S. P. Corgnati, and M. G. Silva, "Assessment of indoor air quality and thermal comfort in Portuguese secondary classrooms: Methodology and results," Building and Environment, vol. 81, pp. 69-80, 2014.

[5] Q. Zhu, J. Yi, S. Sheng, C. Wen, and H. Hu, "A computer-aided modeling and measurement system for environmental thermal comfort sensing," IEEE Transactions on Instrumentation and Measurement, vol. 64(2), pp. 478-486, 2015.

[6] C. Lin, "Forecasting indoor environment using ensemble-based data assimilation algorithms," Ph.D. dissertation, Concordia University, Montreal, Quebec, Canada, 2014.

[7] H. Fang, R. Sharma, and R. Patil, "Optimal sensor and actuator deployment for hvac control system design," in Proc. Conference on American Control Conference, Oregon, USA, June 2014, pp. 22402246.

[8] D. Brunelli, I. Minakov, R. Passerone, and M. Rossi, "POVOMON: An ad-hoc wireless sensor network for indoor environmental monitoring," in Proc. IEEE Workshop on Environmental Energy and Structural Monitoring Systems, Naples, Italy, September 2014, pp. 1-6.

[9] J. Yun and J. Kim, "Deployment support for sensor networks in indoor climate monitoring," International Journal of Distributed Sensor Networks, pp. 1-10, 2013.

[10] N. Cressie and C. K. Wikle, Statistics for spatio-temporal data. Wiley, 2011.

[11] L. V. Nguyen, S. Kodagoda, R. Ranasinghe, and G. Dissanayake, "Information-driven adaptive sampling strategy for mobile robotic wireless sensor network," IEEE Transaction on Control Systems Technology, vol. 24(1), pp. 372-379, 2016.

[12] C. Ko, J. Lee, and M. Queyranne, "An exact algorithm for maximum entropy sampling," Operations Research, vol. 43, pp. 684-691, 1995. 